\title{
Greater ethnic diversity correlates with lower HIV prevalence in Africa: justification for an alloimmunity vaccine
}

This article was published in the following Dove Press journal:

HIVIAIDS - Research and Palliative Care

II April 2013

Number of times this article has been viewed

\section{Christopher Zamani \\ Jared D Elzey \\ James EK Hildreth}

Center for AIDS Health Disparities Research at Meharry Medical College, Nashville, Tennessee, USA
Correspondence: James EK Hildreth College of Biological Sciences, University of California, Davis, One Shields Avenue, Davis, CA 95616, USA

Tel +l 5307524460

Email jekhildreth@ucdavis.edu
Purpose: After decades of research, AIDS continues to be a major pandemic and to date, adaptive immunity vaccine designs have had little to no success. Data indicate the alloimmune response is a potent mitigator of human immunodeficiency virus (HIV) infection, for which experiments of nature should be demonstrable to justify pursuit of an alloimmune vaccine strategy. We sought to determine if large-scale alloimmune diversity correlates with lower HIV infection rates.

Methods: Using published data of African linguistic groups to determine sub-Saharan country ethnicity profiles as a proxy for human leukocyte antigen (HLA) diversity, a correlation analysis was performed against respective sub-Saharan country HIV infection rates. Ethnicity data from 37 subSaharan nations in 2003 and from 38 nations in 2005 were used to calculate the Meyers-Macintosh ethnic diversity score for each nation as the independent variable. World Health Organization data on HIV infection rates for the same countries were used as the dependent variable. The main outcome measure was the correlation coefficient of ethnic diversity versus HIV infection rate.

Results: A significant negative correlation was shown between ethnic diversity and HIV infection: for 2003 data, -0.4586 (two-tailed $P$-value of 0.0043 ); and, for 2005 data, -0.3866 (two-tailed $P$-value of 0.0165 ).

Conclusion: In conjunction with substantial evidence that alloimmunity confers protection against HIV transmission and recent work identifying specific anti-HIV mechanisms, this analysis strongly justifies an HLA-based alloimmune vaccine strategy against HIV.

Keywords: AIDS, adaptive immunity, human leukocyte antigen (HLA)

\section{Introduction}

Decades of funding and research focused on combatting the spread of human immunodeficiency virus (HIV) have produced critical advances in antiretroviral therapies that have extended and improved the lives of those infected with HIV, and have proven effective at reducing transmission to those uninfected when treatment begins early and adherence is maintained. These advances are major biomedical successes, but the advent of an antigenic vaccine has remained elusive. Although a recent HIV-1 subunit vaccine trial in Thailand showed modest preventive effects, ${ }^{1}$ the virus' astonishing ability to hide from and destroy the human immune system through multiple avenues dictates that a traditional vaccine strategy may not be feasible.

Based on multiple lines of evidence and observations, several papers have called for, or cautioned against, development of an alloimmune vaccine strategy (allo-vaccine) against HIV. ${ }^{2-9}$ An allogeneic vaccine would be based roughly on the same mechanism behind organ transplant rejection, human leukocyte antigens (HLAs). HLA serves as a cellular signature that allows differentiation between autologous and nonautologous 
immune response. Every cell in an individual has the same combination of HLA, and because HLA has thousands of variations, it is very rare for two unrelated individuals to have the same HLA signature. As exemplified by organ transplant rejection, the immune system views non-matching or missing HLA as an invasive pathogen and reacts to eliminate the foreign tissue or cells. Potential complications of an alloimmunitybased vaccine include vaccinees being at higher risk of organ rejection after transplantation, dangerous reactions to blood transfusions such as graft-vs-host disease, and complications in pregnancy.

Developing an allo-vaccine presents technical and immunological difficulties, but pursing a traditional vaccine designed to elicit an adaptive immune response to viral proteins is likely to fail, and may even increase susceptibility to HIV. ${ }^{2,10}$ Detailed elsewhere, the Trojan exosome hypothesis of retroviruses proposes that human retroviruses hijack the exosome pathway, a component of intercellular communication. ${ }^{2}$ Exosomes are vesicles released from many cell types, and are coated with functional adhesion molecules and HLA from their parent cells. Recent studies indicate that exosomes are involved in immune function. ${ }^{1-15}$ The HIV envelope is enriched in the same adhesion and HLA molecules as exosomes, ${ }^{16}$ which originally suggested to one of the authors (JEKH) that the virus was usurping the exosome pathway for biogenesis and release. In presenting both viral and host proteins on its membrane, HIV is likely to induce an immune response via viral proteins and subsequently infect responding CD4+ T cells with the aid of the hijacked adhesion molecules. Thus, any vaccine that evokes a CD4+ T cell response may provide the virus with its preferred target.

On initial exposure to HIV, the virus is enveloped with the donor person's HLA signature, giving the recipient's immune system its only opportunity to prevent infection through alloimmunity. If the recipient has lowered immunity from illness or poor nutrition, if the donor HLA signature matches the recipient's closely enough, or just through stochastic effect, the virus may infect a recipient cell and the subsequent viral progeny will now carry the recipient's HLA signature and no alloimmune responses will be invoked. The body's opportunity to stop the initial infection has passed; the immune system of the recipient will now recognize the progeny viruses as autologous with respect to HLA.

\section{Methods}

\section{Rationale}

A number of studies have indicated that alloimmunity can increase resistance to HIV transmission. Alloimmunization in macaques elicits elevated immune responses and decreased simian immunodeficiency virus (SIV) infectivity. ${ }^{17-19}$ HLA class 1 antibody response in macaques has been demonstrated to correlate with protection from SIV infection. ${ }^{19}$ In human studies, alloimmunization confers limited protection against retroviral infection in women. ${ }^{9}$ Other work has suggested that unprotected intercourse with HLA disparate partners might result in a measure of alloimmunization and that mucosal alloimmunization may result in reduced infection by HIV. ${ }^{6}$ Purified whole immunoglobulin G specific to HLA I and II antibodies from women undergoing alloimmunization therapy for recurrent spontaneous abortions has been shown to neutralize HIV in vitro. ${ }^{20}$

Conversely, previous research has also suggested that suppressed cellular alloimmune response may play a role in protection against HIV infection. ${ }^{4}$ However, these investigators later showed that the sex worker cohort studied presents a unique alteration in an inhibitory killer immunoglobulin-like receptor that may lower the threshold for killer cell activation, explaining the low alloimmune response yet persistent HIV negative status of this population. ${ }^{21}$ More recently, Pido-Lopez et al have shown that allostimulation dramatically upregulates $\mathrm{APOBEC} 3 \mathrm{G},{ }^{3}$ a potent antiretroviral cytidine deaminase effective against HIV. ${ }^{22-29}$ Their in vitro findings are complemented with analysis of CD4+ T cells from women undergoing alloimmunization as a therapy against recurrent spontaneous abortion; increased APOBEC3G production in vivo paralleled their in vitro data. In a previous study with the same cohort, other anti-HIV activity associated with the alloimmunization persisted for 12 months. ${ }^{30}$

Closely related individuals, such as siblings, share more HLA similarities than unrelated individuals. HLAs are encoded in DNA; therefore, the greater a population's genetic diversity, the greater their divergence of HLA signatures. Significant diversity in a population's HLA gene pool increases the chances that any two randomly selected individuals will have different HLA signatures. The strength of immune response to foreign HLA correlates directly to its divergence from the host's HLA; thus populations with increasing HLA variety have greater odds of increasingly stronger immune responses to cellular material exchanged during unprotected sex. Accordingly, the Trojan exosome hypothesis predicts that populations with increasing levels of diversity in the HLA gene pool will have decreasing levels of HIV infection, if other transmission factors are controlled for. Simply put, HLA diversity should suppress the spread of HIV in a population. 
If an HLA-dependent alloimmune response is indeed effective against HIV, proof of concept should be demonstrable between HIV discordant partners. Indeed, the degree of concordance at HLA A, B, and DR loci differs significantly between transmitting and nontransmitting serodiscordant couples. ${ }^{31}$ The sharing of HLA B alleles is independently correlated with accelerated intracouple transmission in previously HIV discordant sexual partners. ${ }^{32}$ Additionally, mother-infant concordance at any HLA class I locus is a strong predictor of perinatal transmission..$^{33} \mathrm{We}$ hypothesize that a cocktail of HLAs divergent from those of a geographically defined population can be used as a viable vaccine, consistent with the concept reviewed by Lehner et al. ${ }^{5}$

In the absence of a database of serodiscordant sexual partners and their respective HLA types - a correlational analysis of which would provide compelling epidemiological support or nullification for pursuing a prospective study - we used the Meyer-McIntosh ethnic diversity index ${ }^{34}$ as a proxy for HLA discordance and looked for correlation with HIV prevalence in sub-Saharan African nations.

\section{The Meyer-Mclntosh ethnic diversity index}

The Meyer-McIntosh ethnic diversity index, ${ }^{34}$ created by Phil Meyer of the University of North Carolina and Shawn McIntosh of USA Today in 1991 and used in numerous population studies since, is an equation that produces a coefficient representing ethnic diversity (below). We used this calculation to represent genetic diversity of a population. Various studies have shown that genetic diversity in Africa is closely related to ethno-linguistic groupings. HLA allele DRB1 distribution has been demonstrated to have a high correlation with linguistic differentiations in Africa. ${ }^{35}$ A study on GM and RH haplotype frequencies concluded that language family relationship is the best predictor of genetic relationship in sub-Saharan Africa. ${ }^{36}$ Another study on GM polymorphisms acknowledges that sociocultural differentiation is important to the analysis of genetic diversity on an African continental scale. ${ }^{37}$ Further, it has been demonstrated that self-reported ancestry is a good predictor of genetic make-up. ${ }^{38}$ Accordingly, we believe the Meyer-McIntosh ethnic diversity index score is a sufficient surrogate predictor of HLA diversity in Africa.

\section{Analysis}

Using the index we calculated a diversity coefficient for 38 African nations based on published ethno-linguistic data. ${ }^{39,40}$
This coefficient allows for the relative comparison of ethnolinguistic diversity between nations on a 0 to 1 scale, with 0 representing absolute ethno-linguistic homogeneity and 1 representing absolute heterogeneity. The index calculates the probability that two randomly chosen persons will be members of the same ethnicity: $\mathrm{P}_{\mathrm{E}}=\left(\mathrm{A}^{2}+\mathrm{B}^{2}+\mathrm{C}^{2}+\mathrm{D}^{2}\right)$ where $\mathrm{A}, \mathrm{B}, \mathrm{C}$, and $\mathrm{D}$ are the proportions in the population of four hypothetical ethnic groups. The diversity index is expressed as $1-\mathrm{P}_{\mathrm{E}}$, which represents the likelihood two randomly chosen persons will be of different ethnicities. The number of groups the index can analyze is unlimited, as long as the total of the group proportions equals $100 \%$.

Using World Health Organization HIV data for 2003 and $2005,{ }^{41}$ we compared the rate of HIV positive adults between the ages of 15 and 49 years and the Meyer-McIntosh ethnic diversity score for African nations (37 countries with 2003 data, 38 for 2005). We applied a Pearson correlation with a regression best-fit line and two-tailed regression significance test (Figures 1 and 2). The ethnic diversity data used for the 2003 and 2005 analysis was the same.

\section{Results}

Among the 38 nations examined, ethnic diversity coefficients ranged from $0.2982-0.9740$ (Figure 3). As a result, a correlation coefficient of -0.4586 (two-tailed $P$-value 0.0043 ) was demonstrated between the ethnic diversity index score and adult HIV prevalence in 2003 (Figure 1). For 2005, the same analysis yielded a correlation coefficient of -0.3866 (two-tailed $P$-value 0.0165 , Figure 2).

\section{Discussion}

The negative correlation between ethnic diversity and adult HIV prevalence among 38 African nations supports

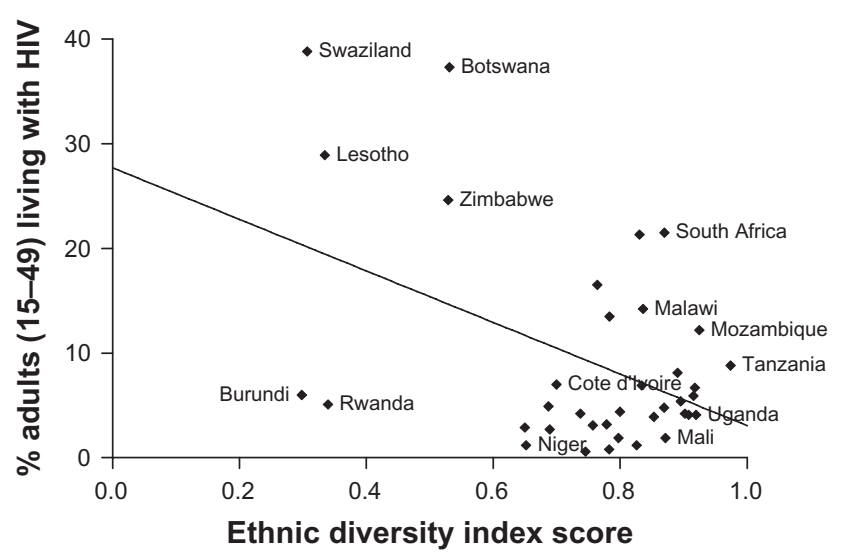

Figure I Correlation between adult HIV prevalence and ethnic diversity in 2003 (select country labels).

Abbreviation: HIV, human immunodeficiency virus. 


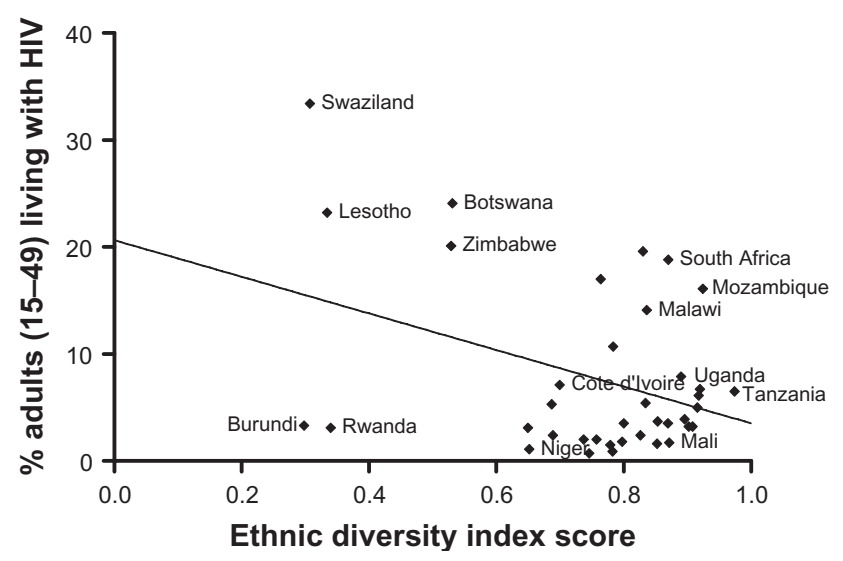

Figure 2 Correlation between adult HIV prevalence and ethnic diversity in 2005 (select country labels).

Abbreviation: HIV, human immunodeficiency virus.

the concept that genetic diversity is protective against HIV transmission. While this correlation analysis cannot determine causality, we feel that in light of emerging and convergent evidence in the literature, these results add weight to the hypothesis that HLA discordance suppresses transmission of HIV, and that an HLA-based vaccine may be a viable prevention strategy. With the previously described research characterizing mechanisms of alloimmunization and its effect on HIV infection, we advocate for the pursuit of an allo-vaccine strategy. We propose that preexposure to divergent HLAs may induce antiretroviral immunity by producing a protective alloimmune response upon natural re-challenge with virus-associated HLA.

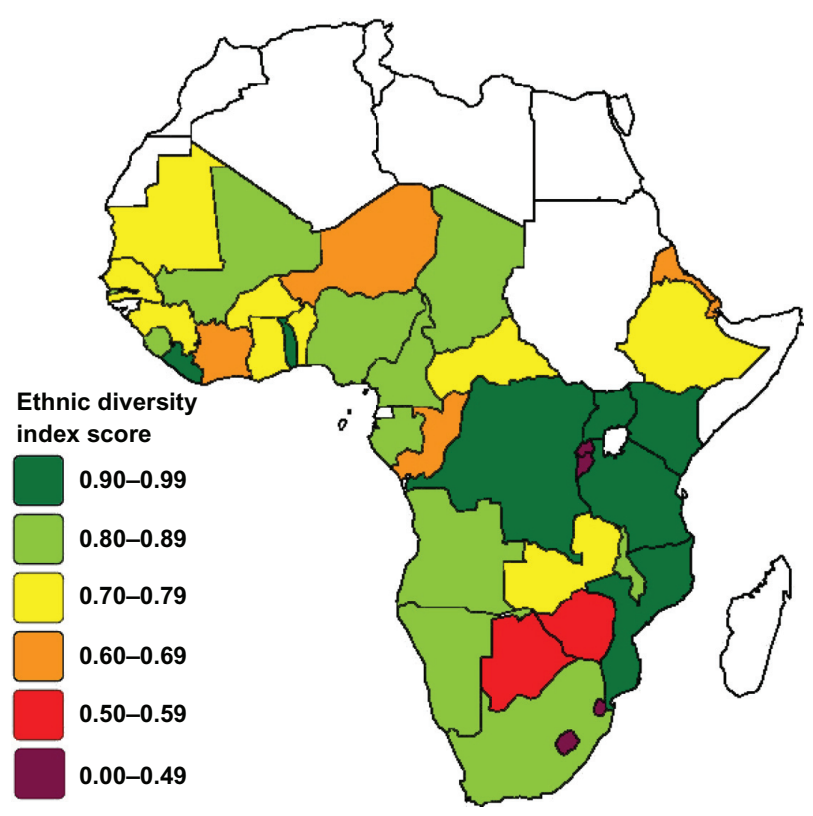

Figure 3 Sub-Saharan African distribution of ethnic diversity index score. Abbreviation: HIV, human immunodeficiency virus.
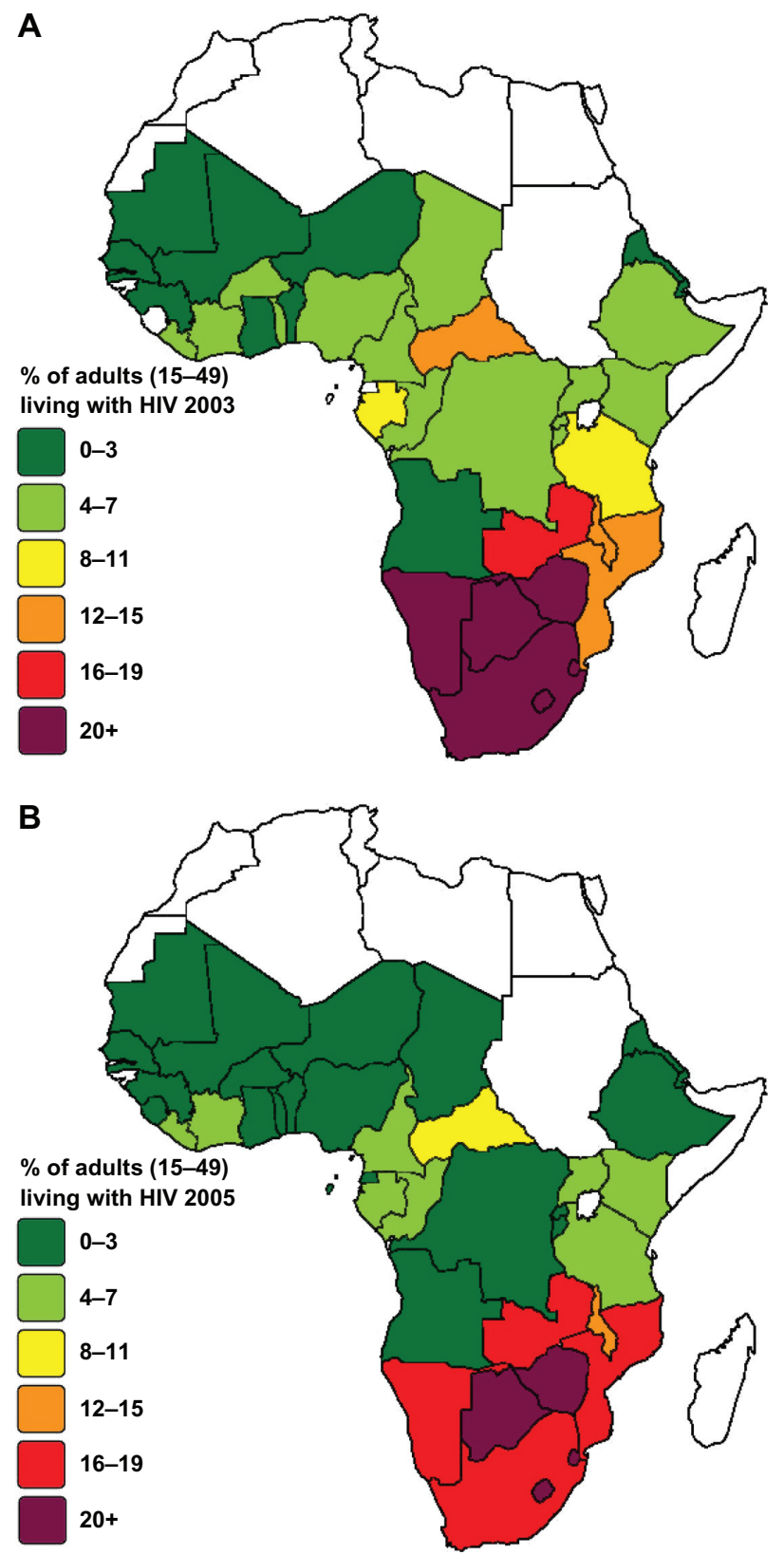

Figure 4 Sub-Saharan African distribution of HIV prevalence. Percent of adults (15-49 years) living with HIV in (A) 2003 and (B) 2005.

Abbreviation: HIV, human immunodeficiency virus. Note: Data from World Health Organization. ${ }^{41}$

There exist some intrinsic limits to the correlation of ethnic diversity with HIV prevalence. Most significant is the imperfect correlation between ethno-linguistic differentiation and HLA diversity at the genetic level, illustrated by Rwanda and Burundi. Both nations have a small ethnic diversity index and a relatively low HIV prevalence that contradicts the predicted inverse correlation (Figures 3 and 4). This may be due to a high level of population flux; regional migration and refugee populations characterize the area due to the longstanding political-military conflicts in Rwanda, Burundi, and eastern 
DR Congo. As the diversity index is based on the indigenous ethnic populations $(n=3)$, it is likely that the genetic diversity of the populations moving through these nations during the 4 decades of conflict is far greater than the indigenous ethnic groups, and has masked an accurate calculation.

Notwithstanding the imperfect proxy of ethnic diversity for HLA distribution, this analysis is compelling from both the strength of the inverse correlation and the continental size of the sample considered. A biologically accurate measure would need to compare infection rates correlated to HLA type among HIV discordant couples. Our hypothesis predicts that in a suitably large sample (to control for confounding variables such as safer-sex practices or antiretroviral therapy use), HLA-divergent couples will remain HIV discordant significantly longer than HLA-similar couples. If such an analysis indicates HLA divergence as protective, then more extensive and focused in vitro and/or animal model research would be justified in pursuit of locale-specific HLA vaccines for HIV high-risk populations.

An HLA vaccine would not be vulnerable to HIV's rapid mutation, as immunity would not be directed against HIVencoded proteins. A strict alloimmune response, primed by a vaccine, will confer little protection against HIV transmission between persons who are highly HLA compatible or HLA identical. However, since APOBEC and other anti-HIV host factors can be upregulated by allostimulation, ${ }^{3,9}$ a meaningful degree of protection may still be conferred.

It must also be noted that vaccines aimed at eliciting the alloimmune response to a broad cocktail of HLA alleles present in a regional population could significantly compromise prospects for organ and tissue transplant among vaccinated individuals. At this point it is not clear that the anti-HIV mechanisms elicited by alloimmunization can be separated from those responsible for allograft rejection. However, fertility clinics have been using lymphocyte immunotherapy, which elicits alloresponses, for decades to treat some forms of infertility issues. For a time, there was controversy surrounding the procedure resulting from concern over a range of potential complications from graft-vs-host disease to autoimmune disorders. Eventually, a large cohort of women $(n=2587)$ was followed for a period of years for adverse effects potentially associated with the therapy. The most frequent complaint was blisters at the intradermal injection site (14\%). None of the theorized negative reactions were reported at greater incidence than would be expected in the general population, and could not be conclusively associated with the treatment. ${ }^{42}$ Thus, the benefits of alloimmunization likely outweigh the risks, particularly in Africa, where the possibility of organ transplant is cost prohibitive, and HIV is quickly devastating millions of lives, creating a generation of orphans, unraveling the social fabric of communities, and devastating national and regional economies.

\section{Conclusion}

Substantial evidence exists that alloimmunity confers protection against HIV transmission. Recent work has identified specific anti-HIV mechanisms. Our analysis showed a significant negative correlation between ethnic diversity (a proxy for genetic diversity) and adult HIV prevalence (a proxy for infectivity) in 37 African nations in 2003 and 38 African nations in 2005. These studies together strongly justify an HLA-based alloimmune vaccine strategy against HIV.

\section{Disclosure/acknowledgments}

Dr Hildreth receives financial support from the United States federal government, the National Institutes of Health (NIH). Dr Hildreth is also a consultant for TM3 Therapeutics LLC, a company developing fully human antibody-based therapeutics and vaccines for human and animal health indications. This work was supported by grant U54 RR019192 to Dr Hildreth from the National Center for Research Resources, a component of the NIH. The others report no other conflicts of interest in this work.

\section{References}

1. Rerks-Ngarm S, Pitisuttithum P, Nitayaphan S, et al. Vaccination with ALVAC and AIDSVAX to Prevent HIV-1 Infection in Thailand. $N$ Engl J Med. 2009;361(23):2209-2220.

2. Gould SJ, Booth AM, Hildreth JE. The Trojan exosome hypothesis. Proc Natl Acad Sci U S A. 2003;100(19):10592-10597.

3. Pido-Lopez J, Wang Y, Seidl T, Babaahmady K, Vaughan R, Lehner T. The effect of allogeneic in vitro stimulation and in vivo immunization on memory CD4(+) T-cell APOBEC3G expression and HIV-1 infectivity. Eur J Immunol. 2009;39(7):1956-1965.

4. Jennes W, Evertse D, Borget MY, et al. Suppressed cellular alloimmune responses in HIV-exposed seronegative female sex workers. Clin Exp Immunol. 2006;143(3):435-444.

5. Lehner T, Shearer GM, Hackett CJ, Schultz A, Sharma OK Alloimmunization as a strategy for vaccine design against HIV/AIDS. AIDS Res Hum Retroviruses. 2000;16(4):309-313.

6. Peters B, Whittall T, Babaahmady K, Gray K, Vaughan R, Lehner T. Effect of heterosexual intercourse on mucosal alloimmunisation and resistance to HIV-1 infection. Lancet. 2004;363(9408):518-524.

7. Quayle AJ, Fidel P Jr, Rosenberg ES. Sex, alloimmunisation, and susceptibility to HIV infection. Lancet. 2004;363(9408):503-504.

8. Shearer GM, Pinto LA, Clerici M. Alloimmunization for immune-based therapy and vaccine design against HIV/AIDS. Immunol Today. 1999; 20(2):66-71.

9. Wang Y, Underwood J, Vaughan R, Harmer A, Doyle C, Lehner T. Allo-immunization elicits CCR5 antibodies, SDF-1 chemokines, and CD8-suppressor factors that inhibit transmission of R5 and X4 HIV-1 in women. Clin Exp Immunol. 2002;129(3):493-501. 
10. Buchbinder SP, Mehrotra DV, Duerr A, et al. Efficacy assessment of a cell-mediated immunity HIV-1 vaccine (the Step Study): a doubleblind, randomised, placebo-controlled, test-of-concept trial. Lancet. 2008;372(9653):1881-1893.

11. Admyre C, Bohle B, Johansson SM, et al. B cell-derived exosomes can present allergen peptides and activate allergen-specific $\mathrm{T}$ cells to proliferate and produce TH2-like cytokines. J Allergy Clin Immunol. 2007;120(6):1418-1424.

12. Admyre C, Johansson SM, Qazi KR, et al. Exosomes with immune modulatory features are present in human breast milk. J Immunol. 2007;179(3):1969-1978.

13. Arita $\mathrm{S}, \mathrm{Baba} \mathrm{E}$, Shibata $\mathrm{Y}$, et al. B cell activation regulates exosomal HLA production. Eur J Immunol. 2008;38(5):1423-1434.

14. Qazi KR, Gehrmann U, Domange Jordö E, Karlsson MC, Gabrielsson S. Antigen-loaded exosomes alone induce Th1-type memory through a B-cell-dependent mechanism. Blood. 2009;113(12):2673-2683.

15. van Niel G, Porto-Carreiro I, Simoes S, Raposo G. Exosomes: a common pathway for a specialized function. J Biochem. 2006;140(1):13-21.

16. Arthur LO, Bess JW Jr, Sowder RC 2nd, et al. Cellular proteins bound to immunodeficiency viruses: implications for pathogenesis and vaccines. Science. 1992;258(5090):1935-1938.

17. Bergmeier LA, Babaahmady K, Wang Y, Lehner T. Mucosal alloimmunization elicits T-cell proliferation, CC chemokines, CCR5 antibodies and inhibition of simian immunodeficiency virus infectivity. J Gen Virol. 2005;86(Pt 8):2231-2238.

18. Bergmeier LA, Walker J, Tao L, Cranage M, Lehner T. Antibodies to human and non-human primate cellular and culture medium components in macaques vaccinated with the simian immunodeficiency virus. Immunology. 1994;83(2):213-220.

19. Stott EJ. Anti-cell antibody in macaques. Nature. 1991;353(6343):393.

20. Leith JG, Clark DA, Matthews TJ, et al. Assessing human alloimmunization as a strategy for inducing HIV type 1 neutralizing anti-HLA responses. AIDS Res Hum Retroviruses. 2003;19(11): 957-965.

21. Jennes W, Verheyden S, Demanet C, et al. Cutting edge: resistance to HIV-1 infection among African female sex workers is associated with inhibitory KIR in the absence of their HLA ligands. $J$ Immunol. 2006;177(10):6588-6592.

22. Alce TM, Popik W. APOBEC3G is incorporated into virus-like particles by a direct interaction with HIV-1 Gag nucleocapsid protein. J Biol Chem. 2004;279(33):34083-34086.

23. Chiu YL, Soros VB, Kreisberg JF, Stopak K, Yonemoto W, Greene WC. Cellular APOBEC3G restricts HIV-1 infection in resting CD4+ T cells. Nature. 2005;435(7038):108-114.

24. Esnault C, Heidmann O, Delebecque F, et al. APOBEC3G cytidine deaminase inhibits retrotransposition of endogenous retroviruses. Nature. 2005;433(7024):430-433.

25. Khatua AK, Taylor HE, Hildreth JE, Popik W. Exosomes packaging APOBEC3G confer human immunodeficiency virus resistance to recipient cells. J Virol. 2009;83(2):512-521.
26. Miyagi E, Opi S, Takeuchi H, et al. Enzymatically active APOBEC3G is required for efficient inhibition of human immunodeficiency virus type 1. J Virol. 2007;81(24):13346-13353.

27. Newman EN, Holmes RK, Craig HM, et al. Antiviral function of APOBEC $3 \mathrm{G}$ can be dissociated from cytidine deaminase activity. Curr Biol. 2005;15(2):166-170.

28. Pion M, Granelli-Piperno A, Mangeat B, et al. APOBEC3G/3F mediates intrinsic resistance of monocyte-derived dendritic cells to HIV-1 infection. J Exp Med. 2006;203(13):2887-2893.

29. Strebel K. APOBEC3G and HTLV-1: inhibition without deamination. Retrovirology. 2005;2:37.

30. Wang Y, Tao L, Mitchell E, et al. Allo-immunization elicits CD8+ T cell-derived chemokines, HIV suppressor factors and resistance to HIV infection in women. Nat Med. 1999;5(9):1004-1009.

31. Lockett SF, Robertson JR, Brettle RP, Yap PL, Middleton D, Leigh Brown AJ. Mismatched human leukocyte antigen alleles protect against heterosexual HIV transmission. J Acquir Immune Defic Syndr. 2001;27(3):277-280.

32. Dorak MT, Tang J, Penman-Aguilar A, et al. Transmission of HIV-1 and HLA-B allele-sharing within serodiscordant heterosexual Zambian couples. Lancet. 2004;363(9427):2137-2139.

33. Polycarpou A, Ntais C, Korber BT, et al. Association between maternal and infant class I and II HLA alleles and of their concordance with the risk of perinatal HIV type 1 transmission. AIDS Res Hum Retroviruses. 2002;18(11):741-746.

34. Meyer P, McIntosh S. The USA Today index of ethnic diversity. Int J Public Opin Res. 1992;4(1):51-58.

35. Sanchez-Mazas A. African diversity from the HLA point of view: influence of genetic drift, geography, linguistics, and natural selection. Hum Immunol. 2001;62(9):937-948.

36. Excoffier L, Harding RM, Sokal RR, Pellegrini B, Sanchez-Mazas A. Spatial differentiation of RH and GM haplotype frequencies in SubSaharan Africa and its relation to linguistic affinities. Hum Biol. 1991;63(3):273-307.

37. Blanc M, Sanchez-Mazas A, Van Blyenburgh NH, Sevin A, Pison G, Langaney A. Interethnic genetic differentiation: GM polymorphism in eastern Senegal. Am J Hum Genet. 1990;46(2):383-392.

38. Excoffier L. Human diversity: our genes tell where we live. Curr Biol. 2003;13(4):R134-R136.

39. World Statesmen.org [homepage on the Internet]. Ben M Cahoon; 2013. Available from: http://www.worldstatesmen.org. Accessed August 7, 2012 .

40. Yakan MZ. Almanac of African Peoples and Nations. New Brunswick, NJ: Transaction Publishers; 1999.

41. www.who.int [homepage on the internet]. Geneva, Switzerland: World Health Organization; 2013. Available from: http://apps.who.int/gho/ data/\#. Accessed January 14, 2010.

42. Kling C, Steinmann J, Westphal E, Magez J, Kabelitz D. Adverse effects of intradermal allogeneic lymphocyte immunotherapy: acute reactions and role of autoimmunity. Hum Reprod. 2006;21(2):429-435.
HIV/AIDS - Research and Palliative Care

\section{Publish your work in this journal}

HIV/AIDS - Research and Palliative Care is an international, peerreviewed open-access journal focusing on advances in research in HIV, its clinical progression and management options including antiviral treatment, palliative care and public healthcare policies to control viral spread. The journal welcomes original research, basic science,

\section{Dovepress}

clinical \& epidemiological studies, reviews \& evaluations, expert opinion \& commentary, case reports \& extended reports. The manuscript management system is completely online and includes a very quick and fair peer-review system. Visit http://www.dovepress.com/ testimonials.php to read real quotes from published authors. 